\title{
MultiColor imaging to detect different subtypes of retinal microaneurysms in diabetic retinopathy
}

\author{
Alessandro Arrigo $\mathbb{i}^{1} \cdot$ Michel Teussink ${ }^{2} \cdot$ Emanuela Aragona $^{1} \cdot$ Francesco Bandello $^{1}$ - Maurizio Battaglia Parodi ${ }^{1}$
}

Received: 30 July 2019 / Revised: 1 November 2019 / Accepted: 2 December 2019 / Published online: 17 February 2020

(c) The Author(s), under exclusive licence to The Royal College of Ophthalmologists 2020

\begin{abstract}
Background Retinal microaneurysms (MAs) are among the earliest signs of diabetic retinopathy (DR) and are typically detected by fluorescein angiography (FA). Confocal MultiColor is a noninvasive-imaging technique able to analyze different retinal features by capturing three simultaneous reflectance images. The main aim of the present study was to characterize morphological features of MAs by means of MultiColor images and to compare these with spectral domain optical coherence tomography (SD-OCT) and FA findings.

Methods A cross-sectional, observational study setting was chosen. Multimodal imaging included MultiColor, SD-OCT and FA images. We performed a qualitative analysis in order to assess the relationship between MultiColor and its green- and red-reflectance components, SD-OCT (hyperreflective, hyporeflective and mixed reflectivity) and FA findings. MAs detected on our MultiColor images were then categorized in accordance with a previously published histological classification.

Results In our study FA images were used to detect 153 MAs in 30 eyes displaying DR. MultiColor was able to distinguish 122 MAs (80\%). We identified green (16\%), red (19\%), and mixed (65\%) MAs, corresponding to different reflectivity features detected by SD-OCT. MAs not visualized on MultiColor images corresponded to tiny hyperreflective lesions on SD-OCT. We compared our imaging findings with a histological MA classification reported in the literature. Our findings showed a strict relationship between MA subtypes and SD-OCT, suggesting that the composition of MAs (cells + endothelium + fibrosis) may influence the signal detected in MultiColor images.

Conclusions MultiColor appears to be a useful technique for investigating MA features in patients with DR.
\end{abstract}

\section{Introduction}

Retinal microaneurysms (MAs) are among the earliest clinical signs of diabetic retinopathy (DR) [1]. Fluorescein angiography (FA) is the gold standard for the proper detection of MAs, whose shapes fall into various categories: focal bulge, saccular, fusiform, mixed, pedunculated, and irregular [2]. The importance of MAs is not merely of a descriptive nature; their turnover correlates with the onset of diabetic macular edema and visual acuity loss [3]. Confocal MultiColor is a non-invasive imaging modality that manages to easily capture three simultaneous reflectance images

Alessandro Arrigo

alessandro.arrigo@hotmail.com

1 Department of Ophthalmology, Scientific Institute San Raffaele, Vita-Salute University, Milan, Italy

2 Heidelberg Engineering GmbH, Heidelberg, Germany using monochromatic blue (wavelength, $486 \mathrm{~nm}$ ), green $(518 \mathrm{~nm})$ and infrared $(815 \mathrm{~nm})$ light [4]. The longer the wavelength, the deeper the light penetrates into the retinal structure, while shorter wavelengths provide a higher contrast of superficial structures, thus enabling different retinal features to be analyzed [4]. The main aim of the present study is to characterize the morphological features of MAs by means of MultiColor images and to compare these with spectral domain optical coherence tomography (SD-OCT) and FA findings.

\section{Methods}

This is a cross-sectional, observational study. Patients affected by type 2 diabetes were recruited at the Ophthalmology Unit of San Raffaele Hospital, Milan, Italy. All patients gave written informed consent. The entire study was conducted in accordance with the Declaration of Helsinki and was approved by the Ethical Committee of the 
Vita-Salute San Raffaele University in Milan. Patients with a confirmed diagnosis of DR, based on a diagnosis of any stage of DR and clear funduscopic detection of MAs in the posterior pole, were included. Patients with significant media opacity, any other type of retinal or optic nerve disease, ocular surgery within the last 3 months, or with any systemic condition that could affect these analyses were excluded from the study.

All patients underwent a complete ophthalmic examination, including ETDRS best-corrected visual acuity (BCVA, expressed as the logarithm of the minimal angle or resolution), applanation tonometry, and anterior, and posterior segment slit lamp evaluation.

Multimodal imaging included MultiColor, blue autofluorescence (BAF), infrared (IR), SD-OCT, and FA images, acquired by means of a Spectralis HRA+OCT device (Heidelberg Engineering, Heidelberg, Germany). SD-OCT acquisitions included raster, radial, and dense scans with a high number of frames (ART $>25$ ) and enhanced depth imaging. FA images were analyzed at early, intermediate and late phases of the dye transit. Highly averaged MultiColor images were acquired using a large number of frames $(>70)$, in order to obtain high quality data.

Based on the acquired images, we performed a qualitative analysis in order to assess the relationship between MultiColor and its green- and red-reflectance components, SD-OCT (hyperreflective, hyporeflective and mixed reflectivity) and FA findings. MA wall thickness was arbitrarily evaluated on the basis of the hyperreflectivity of the border surrounding the MA space. The agreement between the findings provided by each technique was also assessed. We categorized MAs detected on our MultiColor images in accordance with the histological classification provided by Stitt and colleagues [5]. Finally, we compared MA detection as achieved by MultiColor, BAF and IR images.

\section{Results}

Our study included 153 MAs of 30 eyes (30 DR patients; mean age $55 \pm 9 ; 17$ males) detected at the posterior pole in FA images. On FA, MAs fill with dye and display different degrees of perilesional leakage. Eighty percent of these MAs (122 MAs) were detected on MultiColor images and were included in the analysis. Mean BCVA was $0.6 \pm 0.2$ LogMAR. Ten eyes were affected by diabetic macular edema. We identified three different MA types on MultiColor images. Green MAs corresponded mainly to a hyperreflective signal on SD-OCT; red MAs were characterized by a hyperreflective margin and an unevenly reflective core on SD-OCT. We also identified mixed red and green MAs on MultiColor, with a hyperreflective border and hyporeflective core on SD-OCT (Fig. 1). No marked FA differences were found between the various red, green, and mixed MAs. Green MAs showed both poor filling and poor leakage phenomena; red and mixed MAs both showed full filling and variable leakage phenomena, although these were more pronounced for mixed MAs (Fig. 1). We found a strong correspondence between the thickness of the MA wall on MultiColor and wall reflectivity properties on SD-OCT (both defined as thin, thick, or intermediate), as well as a strong correspondence regarding MA content detected by both techniques (Fig. 2). No exceptions were observed regarding both these qualitative agreements between MultiColor and SD-OCT findings.

Of all MAs analyzed, $16 \%$ were classified as green, $19 \%$ as red, and $65 \%$ as mixed. We arbitrarily interpreted our MultiColor and SD-OCT findings as described in Table 1, comparing them with the histological classification of MAs, involving four different types of lesion. MAs identified on FA were not detectable on MultiColor imaging in $20 \%$ of cases. SD-OCT inspection of these MAs typically revealed tiny, hyperreflective lesions (Fig. 3). Comparative analysis of MultiColor, BAF, and IR images showed $80 \%$ agreement. In particular, $20 \%$ of MAs were found to have been missed in IR and BAF owing to the presence of edemas, hemorrhages, or other types of interference. Moreover, BAF and IR images provided less information regarding MA content and wall features than MultiColor ones (Fig. 4).

\section{Discussion}

The present study describes three different types of MA based on MultiColor images: red, green and mixed. These corresponded to differently defined findings on structural OCT. However, FA did not show marked differences in terms of filling, staining, and leaking phenomena.

Our interpretation of the different MA colors in the MultiColor findings was strictly based on the extent of fibrotic changes associated with MAs. In particular, a greater degree of fibrosis correlates with a stronger green reflectance signal on MultiColor. In addition, the amount of cellular/extracellular material inside MA correlates with the intensity of near-infrared reflectance on MultiColor. Indeed, the association between fibrotic changes and increased green reflectance on MultiColor has been described previously [4]. Our SD-OCT findings lend further support to the above associations. Specifically, we found that the thickness of the MA wall detected on MultiColor, and the intensity of the green reflectance related to the fibrotic degeneration matched the wall reflectivity properties on SD-OCT very well. An equally good match was found concerning the MA content detected by both imaging techniques. Our interpretation 

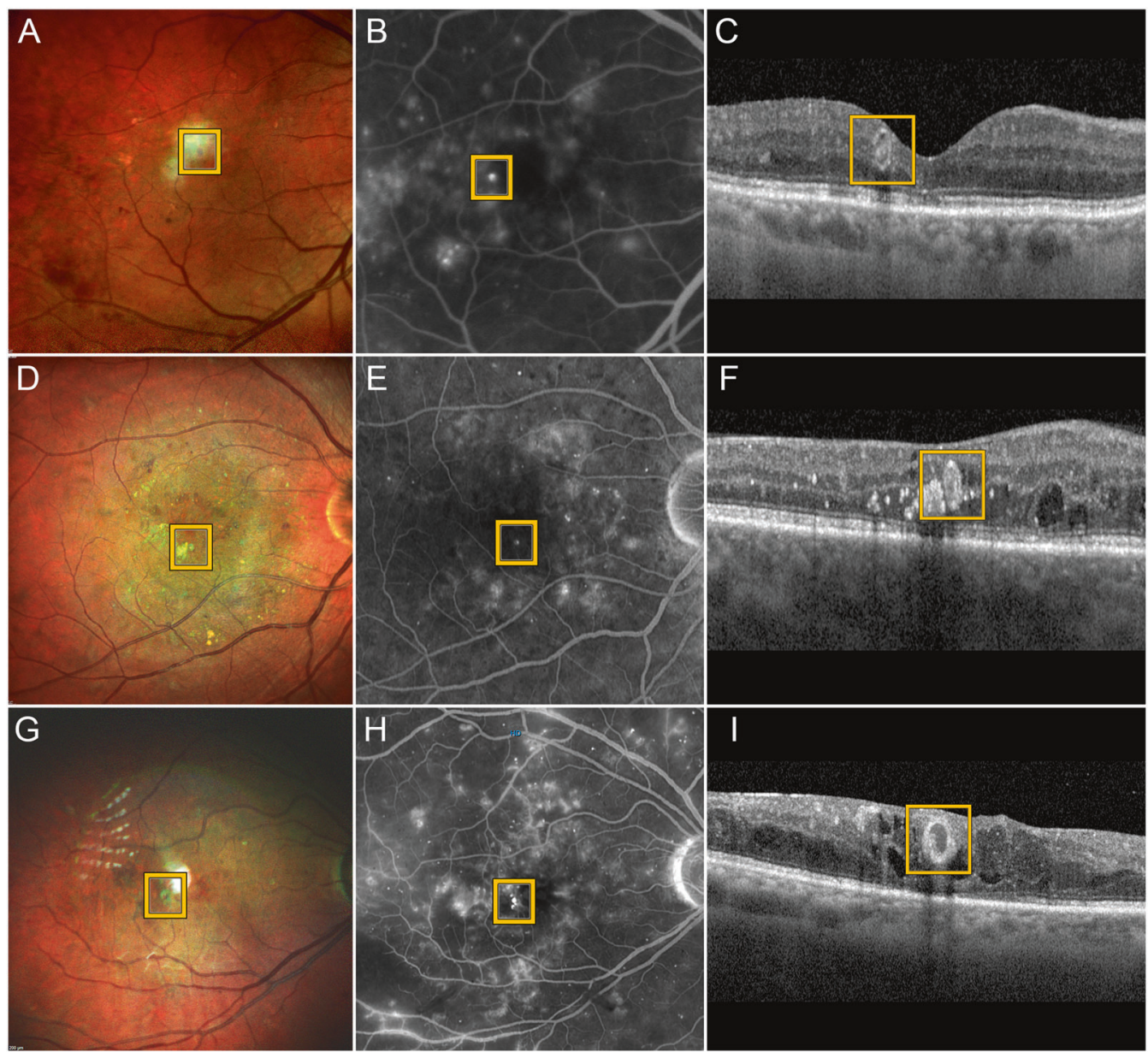

Fig. 1 Multicolor imaging in retinal microaneurysms. Red MAs (a) are characterized by full filling and variable leakage phenomena on FA (b), corresponding to a hyperreflective margin and unevenly reflective core on structural OCT (c). Green MAs (d) show less pronounced filling and leakage on FA (e) and are detected as mainly hyperreflective on structural OCT (f). Mixed MAs (g) show good filling and also more pronounced leakage on FA (h); on structural OCT they display a hyperreflective border and hyporeflective core (i). also found support in the excellent histological MA classification provided by Stitt et al. [5]. In particular, they reported that MAs can be diversely affected by the loss of endothelial cells and pericytes, as well as by lipid infarction and fibrosis. All of these changes are also linked to the number of red blood cells occupying the MA lumen and their loss of integrity.

It may be assumed that these changes occur in no particular order, thus potentially coexisting in different amounts in each MA. This might be the reason why most of our MAs had mixed colors on MultiColor images, and it might also explain why there were no FA changes characteristic of each type of MA. Instead, the filling, staining, and leaking phenomena are strictly related to the amount of perfusion involving the MAs, as are their degenerative changes. Moreover, as the composition of MAs (cells + endothelium + fibrosis) may be extremely diverse, it can be assumed that possible leakage points can be found in all MAs, which significantly complicates efforts to correctly classify MAs based on FA. In addition, FA is known to be affected by resolution limits as well as by diffuse leakage phenomena interfering with the proper analysis of the MA signal. For all these reasons, further investigations should be conducted in order to properly assess the FA features of each different type of MA. It is worth noting the $80 \%$ agreement between MultiColor, BAF, and IR images when detecting MAs, thus demonstrating that MultiColor is more useful than the other two techniques in providing detailed information regarding MA content and wall features.

Collectively, our findings indicate the utility of MultiColor as a noninvasive in vivo imaging technique when investigating the microstructural properties of MAs. Bearing in mind the dynamic behavior of MAs, especially with regard to the strong association between MA turnover and DR severity and progression risk [3], and considering the relationship between internal MA reflectivity and perfusion [6], MultiColor imaging can be regarded as 
a useful and easily repeatable way of performing a first level investigation and follow-up of the lesions. Indeed, when used to depict the entire scenario of the posterior

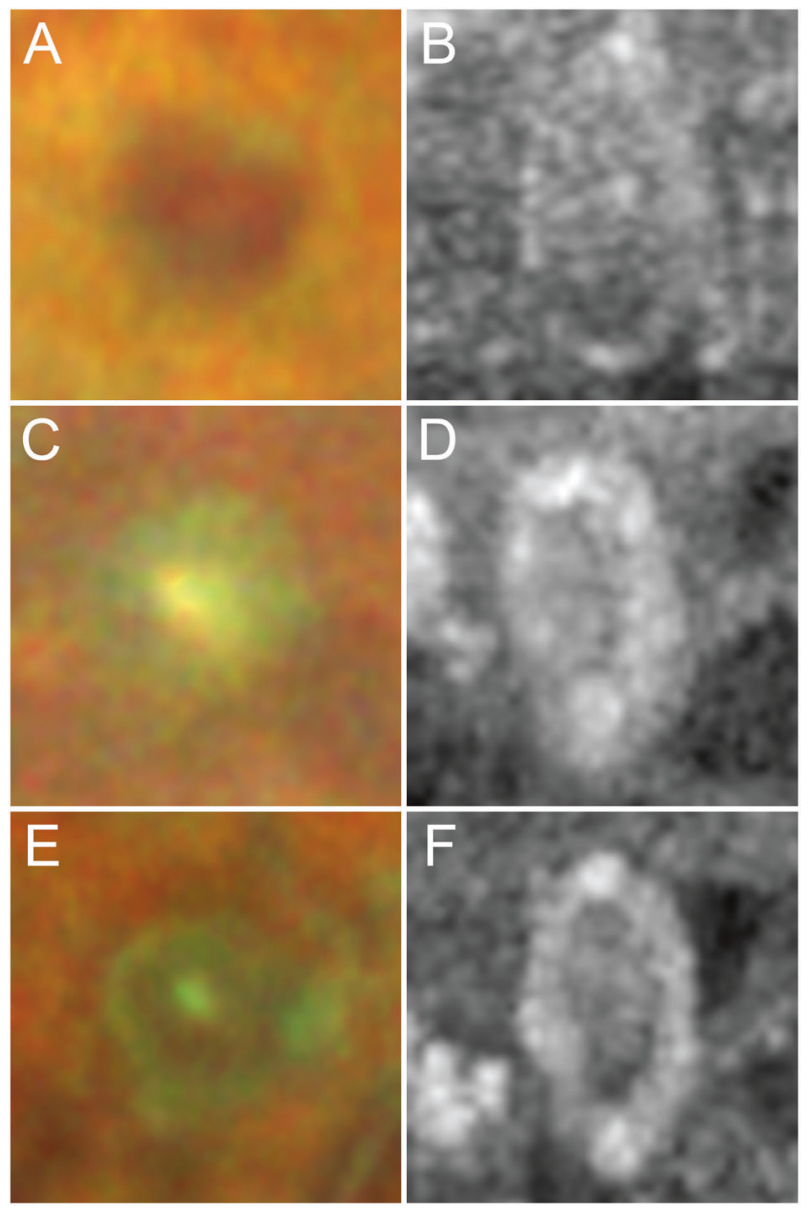

Fig. 2 MultiColor and structural OCT features of retinal microaneurysms. The techniques match well when describing both MA content and wall-reflectivity properties. In particular, red MAs are shown in a, b, green MAs are shown in $\mathbf{c}$, $\mathbf{d}$, and mixed MAs are shown in e, f. MA wall thickness was judged as thin $(\mathbf{a}, \mathbf{b})$, thick $(\mathbf{c}, \mathbf{d})$, and intermediate $(\mathbf{e}, \mathbf{f})$. pole, MultiColor imaging is less time consuming and less stressful for patients than SD-OCT, which requires longer acquisitions.

Our study has undoubted limitations, especially with regard to possible imaging artefacts. More extensive future studies should also include SD-OCT angiography investigations, in order to assess the relationship between retinal blood perfusion and MultiColor findings. We should add that we decided not to conduct a histological analysis of the same eyes, preferring to compare our data with a previous histological classification. Further studies are needed to effectively assess the correspondence between MA histology and subtypes based on MultiColor analysis.

In conclusion, our study reported three different types of MAs related to DR based on MultiColor images. These subtypes showed a strict relationship with SD-OCT findings, suggesting that the number of fibrotic changes may influence MA color in MultiColor images. MultiColor appears to be a useful and repeatable technique to perform a first-level investigation of MAs at baseline and during the follow-up of patients with DR.

\section{Summary}

\section{What was known before}

- Retinal microaneurysms are of the earliest signs characterizing diabetic retinopathy and are typically detected by fluorescein angiography.

\section{What this study adds}

- In the present study, we described three different types of microaneurysms based on MultiColor images,

Table 1 Microaneurysms Classification.

\begin{tabular}{llll}
\hline Microaneurysms classification & MultiColor & Structural OCT \\
\hline Histologic classification & $\begin{array}{l}\text { Extensive accumulation of polymorphonuclear } \\
\text { cells into the lumen, with intact endothelium } \\
\text { and absent pericytes. }\end{array}$ & $\begin{array}{l}\text { Hyperreflective margin and unevenly } \\
\text { reflective core. }\end{array}$ \\
Type I & $\begin{array}{l}\text { Large numbers of red blood cells in the lumen, with } \\
\text { completely absent endothelial cells and pericytes. }\end{array}$ & Mixed & Hyperreflective border and \\
Type III & $\begin{array}{l}\text { Nonperfused MA, with aggregates of irregularly shaped } \\
\text { red blood cells profiles and breakdown products. }\end{array}$ & Green & Mainly hyperreflective. \\
Type IV & $\begin{array}{l}\text { Almost or completely sclerosed, with extensive } \\
\text { fibrosis and lipid infiltration into the lumen and } \\
\text { basement membrane wall. }\end{array}$ & &
\end{tabular}

MultiColor and structural OCT findings are compared with the histological classification adapted from Stitt et al. [5]. 
Fig. 3 Exemplary case of MA identified on FA (a), but not detectable on MultiColor (b) imaging. Structural OCT typically shows tiny, hyperreflective MAs (c).
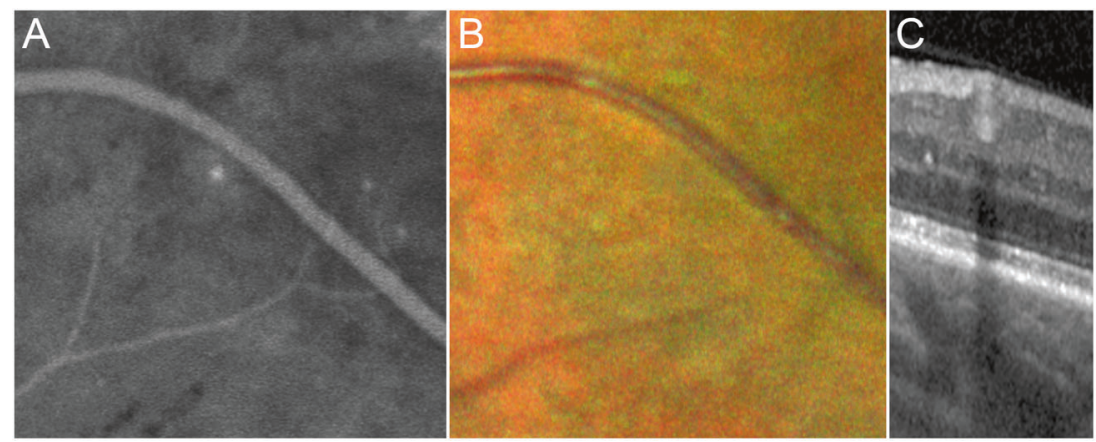
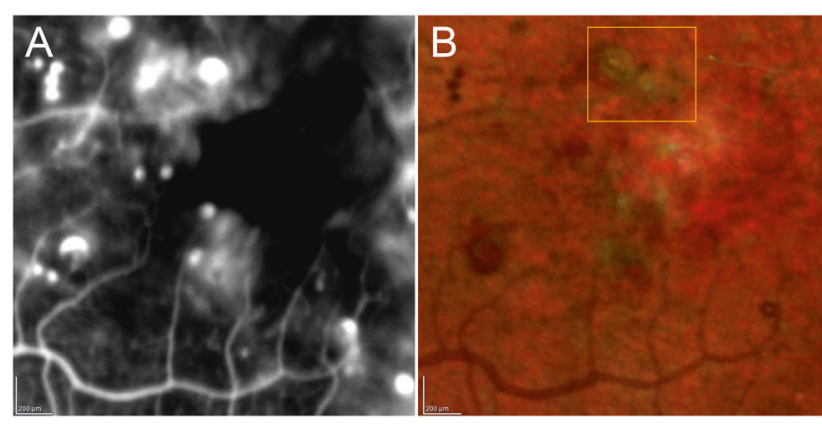

Fig. 4 Comparative MA analysis between MultiColor, IR, and BAF images. Compared with FA (a), MultiColor imaging (b) provided good information regarding MA composition and wall

respectively red, green, and mixed. These corresponded to different defined findings on structural OCT, however, FA did not show marked differences in terms of filling, staining, and leaking phenomena. Microaneurysms colors was based strictly in the context of the extent of fibrotic changes associated with microaneurysms.

Funding FB consultant for: Alcon (Fort Worth, TX, USA), Alimera Sciences (Alpharetta, GA, USA), Allergan Inc (Irvine, CA, USA), Farmila-Thea (Clermont-Ferrand, France), Bayer Shering-Pharma (Berlin, Germany), Bausch and Lomb (Rochester, NY, USA), Genentech (San Francisco, CA, USA), Hoffmann-La-Roche (Basel, Switzerland), NovagaliPharma (Évry, France), Novartis (Basel, Switzerland), Sanofi-Aventis (Paris, France), Thrombogenics (Heverlee, Belgium), Zeiss (Dublin, USA). All other authors have no disclosures to declare.

\section{Compliance with ethical standards}

Conflict of interest The authors declare that they have no conflict of interest.
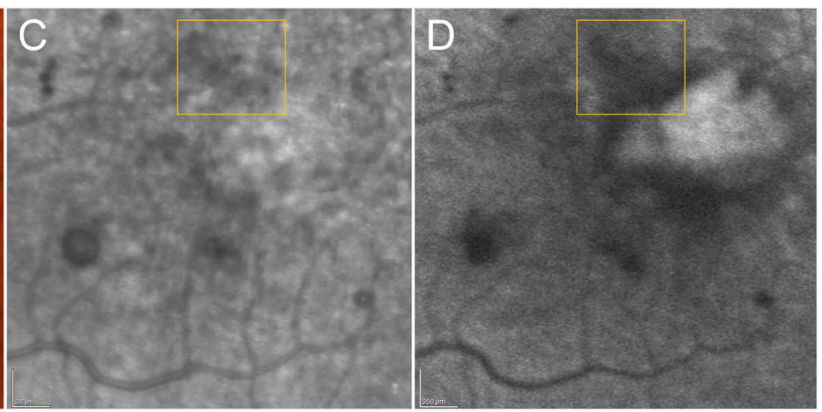

properties. In contrast, both IR (c), and BAF (d) images showed fewer details regarding MAs. It is worth noting the absence of some MAs both in IR and in BAF (orange square).

Publisher's note Springer Nature remains neutral with regard to jurisdictional claims in published maps and institutional affiliations.

\section{References}

1. Frank RN. Diabetic retinopathy. N. Engl J Med. 2004;350:48-58.

2. Dubow M, Pinhas A, Shah N, Cooper RF, Gan A, Gentile RC, et al. Classification of human retinal microaneurysms using adaptive optics scanning light ophthalmoscope fluorescein angiography. Investig Ophthalmol Vis Sci. 2014;55:1299-309.

3. Pappuru RKR, Ribeiro L, Lobo C, Alves D, Cunha-Vaz J. Microaneurysm turnover is a predictor of diabetic retinopathy progression. Br J Ophthalmol. 2019;103:222-6.

4. Tan ACS, Fleckenstein M, Schmitz-Valckenberg S, Holz FG. Clinical application of multicolor imaging technology. Ophthalmologica. 2016;236:8-18.

5. Stitt AW, Gardiner TA, Archer DB. Histological and ultrastructural investigation of retinal microaneurysm development in diabetic patients. Br J Ophthalmol. 1995;79:362-7.

6. Parravano M, De Geronimo D, Scarinci F, Querques L, Virgili G, Simonett JM, et al. Diabetic microaneurysms internal reflectivity on spectral-domain optical coherence tomography and optical coherence tomography angiography detection. Am J Ophthalmol. 2017;179:90-6. 